Mon. Not. R. Astron. Soc. 000, 1-?? () Printed 28 August $2018 \quad$ (MN LATEX style file v2.2)

\title{
Vortex formation in protoplanetary discs induced by the vertical shear instability
}

\author{
Samuel Richard ${ }^{1 \star}$, Richard P. Nelson ${ }^{1}$ \& Orkan M. Umurhan ${ }^{2,3}$ \\ 1 Astronomy Unit, Queen Mary University of London, Mile End Rd, London, E1 4NS, U.K. \\ 2 Space Sciences Division, NASA Ames Research Center, Moffett Field, CA 94035, USA \\ 3 SETI Institute, 189 Bernardo Way, Mountain View, CA 94043, USA
}

\begin{abstract}
We present the results of $2 \mathrm{D}$ and $3 \mathrm{D}$ hydrodynamic simulations of idealized protoplanetary discs that examine the formation and evolution of vortices by the vertical shear instability (VSI). In agreement with recent work, we find that discs with radially decreasing temperature profiles and short thermal relaxation time-scales, are subject to the axisymmetric VSI. In three dimensions, the resulting velocity perturbations give rise to quasi-axisymmetric potential vorticity perturbations that break-up into discrete vortices, in a manner that is reminiscent of the Rossby wave instability. Discs with very short thermal evolution time-scales (i.e. $\tau \leqslant 0.1$ local orbit periods) develop strong vorticity perturbations that roll up into vortices that have small aspect ratios $(\chi \leqslant 2)$ and short lifetimes ( $\sim$ a few orbits). Longer thermal time-scales give rise to vortices with larger aspect ratios $(6 \leqslant \chi \leqslant 10)$, and lifetimes that depend on the entropy gradient. A steeply decreasing entropy profile leads to vortex lifetimes that exceed the simulation run times of hundreds of orbital periods. Vortex lifetimes in discs with positive or weakly decreasing entropy profiles are much shorter, being 10s of orbits at most, suggesting that the subcritical baroclinic instability plays an important role in sustaining vortices against destruction through the elliptical instability. Applied to the outer regions of protoplanetary discs, where the VSI is most likely to occur, our results suggest that vortices formed by the VSI are likely to be short lived structures.
\end{abstract}

Key words: accretion, accretion discs - hydrodynamics - instabilities - protoplanetary discs - turbulence

\section{INTRODUCTION}

The presence of long-lived vortices in protoplanetary discs has long been considered as a means of enhancing the planet building process because of the ability of these anticyclonic structures to capture and concentrate dust grains (von Weizsäcker 1944, Barge \& Sommeria 1995; Johansen et al. 2004). The presence of vortices in discs can also lead to significant transport of angular momentum through the excitation of spiral density waves (Johnson \& Gammie 2005). In spite of the potential importance of vortices for the evolution of protoplanetary discs and planet formation, however, the following questions do not yet have definitive answers: If vortices exist in protoplanetary discs, what are their formation mechanisms?; Which regions of protoplanetary discs can support the existence of vortices?; What are the lifetimes of vortices in protoplanetary discs?

* E-mail: samuel.richard@qmul.ac.uk; r.p.nelson@qmul.ac.uk; orkan.m.umurhan@nasa.gov
A number of hydrodynamic instabilities have been suggested as vortex formation mechanisms. The Rossby wave instability (RWI; Lovelace et al. 1999, Li et al. 2000, 2001) has been shown to produce large scale vortices from an initial axisymmetric state when a non self-gravitating disc has a sufficiently strong local minimum in the potential vorticity (Umurhan 2010, Lovelace \& Hohlfeld 2013, Yellin-Bergovoy et al. 2015). The extremum can be due to a local maximum in surface density, such as can appear at the edge of the dead zone where a sharp change in effective disc viscosity arises (e.g. Varnière \& Tagger 2006), or because a planet opens a gap in the disc (de Val-Borro et al. 2007). Another way to produce vortices is through baroclinic instability. Klahr \& Bodenheimer (2003) introduced the concept of the global baroclinic instability (GBI), and suggested that a disc with a global negative entropy gradient could form vortices. More recently, Petersen et al. (2007) and Petersen et al. (2007) have shown that vortices can form when the disc has an unstable radial stratification and undergoes thermal relaxation (or 'cooling'). Lesur \& Papaloizou (2010) showed in 
their study that this instability is actually a nonlinear instability (requiring finite amplitude perturbations to be activated) and called it the subcritical baroclinic instability (SBI). The nonlinearity of the instability means that the finite amplitude perturbations need to be generated by another unspecified process. A linear instability that has been suggested as a possible source for these perturbations is the 'convective overstability', in which the growth of epicyclic oscillations is powered by the same unstable stratification and thermal relaxation required for the subcritical instability, leading to the formation of long-lived vortices (Klahr \& Hubbard 2014 Lyra 2014). Finally, there have been recent suggestions that a zombie vortex instability may arise in stably stratified flows by the formation of a critical layer which rolls up into vortices, which then excite new critical layers and vortices, leading eventually to space filling turbulence that is dominated by large vortices (Marcus et al. 2013, 2015).

In this paper, we examine the possibility that vortices may be formed by the vertical shear instability (VSI). This is a linear instability that was first studied by Goldreich \& Schubert (1967) and Fricke (1968) in the context of differentially rotating stars. The presence of vertical shear in protoplanetary discs must arise when there is a radial temperature gradient, and this can lead to the destabilization of inertial-gravity waves (oscillations for which rotation and buoyancy provide restoring forces) when thermal time-scales are shorter than viscous time-scales, and are of the order of, or shorter than, dynamical time-scales Urpin \& Brandenburg 1998, Urpin 2003, Nelson et al. 2013; Barker \& Latter 2015 Umurhan et al. 2015). The recent study by Nelson et al. (2013) adopted simple equations of state and cooling prescriptions, and showed that this instability can lead to sustained hydrodynamic turbulence with a Shakura Sunyaev angular momentum transport parameter $\alpha \sim 10^{-3}$ for very short cooling times. Stoll \& Kley (2014) performed nonlinear hydrodynamic simulations with radiation transport and showed that the instability operates in the presence of a more complete description of the gas thermal evolution, albeit with a reduced efficiency of angular momentum transport. The requirement for very short cooling times suggests that this instability is most likely to operate in the outer regions of protoplanetary discs beyond $\sim 10$ au (Nelson et al. 2013 Umurhan et al. 2013). An analysis of linear growth rates in discs with energy transport in both the optically thick and thin regimes presented by Lin \& Youdin (2015) suggests that the VSI should operate at radii in the range $1050 \mathrm{au}$.

This paper is organized as follows. In Sect.2, we review the different processes and instabilities that can lead to the formation and destruction of vortices in discs, and in Sect. 3 we describe the disc models that are the basis of our study and the numerical scheme used in the simulations. The results of two-dimensional, axisymmetric simulations are presented in Sect. 4, and the results of three-dimensional runs that examine the formation and evolution of vortices are presented in Sect. 5. Finally we discuss our results and draw conclusions in Sect. 6

\section{THEORETICAL BACKGROUND AND EXPECTATIONS}

Before presenting our simulation results we discuss a number of theoretical results that are of relevance to this numerical study of the VSI. We make use of both spherical $(r, \theta, \phi)$ and cylindrical $(R, \phi, Z)$ coordinates in this paper. The cylindrical coordinates are used in the formulae giving the disc structure (density, temperature and velocity profile) for convenience, while the spherical coordinates are used for the simulations because they fit better with the shapes of the disc models.

\section{$2.1 \quad$ RWI}

The first vortex-forming instability that has been studied in the context of protoplanetary discs with near-Keplerian rotation profiles is the RWI. Using a combination of linear analysis and nonlinear numerical simulations, Lovelace et al. (1999), Li et al. (2000) and Li et al. (2001) showed that a non-axisymmetric instability may develop, leading to the formation of a number of vortices, when the disc contains a local extremum in the function:

$\mathcal{L}=\frac{\Sigma}{\omega_{z}}\left(\frac{P}{\Sigma \gamma}\right)^{2 / \gamma}$

where $\omega_{z}$ is the vertical component of the vorticity, $\Sigma$ is the surface density and $P$ is the pressure $\left(\omega_{z} / \Sigma\right.$ is the potential vorticity and $P / \Sigma^{\gamma}$ is related to the entropy). It has been observed that these vortices often merge to form a single vortex during the advanced stages of nonlinear evolution. Considered within the context of protoplanetary discs, the RWI is normally observed to develop when a local pressure or density maximum is present in the disc, such as may occur at the edge of a planet-induced gap (de Val-Borro et al. 2006 2007) or at the edge of a dead zone where there is a sharp transition in the disc viscosity (Lyra \& Mac Low 2012 ). Vortices have also been observed to develop spontaneously in global magnetized disc models that sustain the magnetorotational instability (Fromang \& Nelson 2005). Although this latter phenomenon has not been explored in detail, a possible explanation is that the vortices arise because the RWI feeds off the so-called zonal flows that are observed to arise in discs with MHD turbulence (Steinacker \& Papaloizou 2002, Papaloizou \& Nelson 2003 |Johansen et al. 2009 Bai \& Stone 2014).

\subsection{SBI}

The existence of a vortex-forming baroclinic instability operating in protoplanetary discs was first suggested by Klahr \& Bodenheimer (2003), based on a series of nonlinear simulations conducted using disc models with negative radial entropy gradients. The linear properties of this GBI were investigated by Klahr (2004) and Johnson \& Gammie (2005), who found evidence for only transient growth. The nonlinear evolution was investigated using shearing box simulations by Johnson \& Gammie (2006), who found no instability. Petersen et al. (2007) examined disc stability using global anelastic simulations of baroclinic discs with prescribed cooling and observed the growth of vortices that were found to survive for hundreds of orbits (Petersen et al. 2007). In a 
subsequent paper, Lesur \& Papaloizou (2010) used both incompressible and compressible shearing box simulations to examine the growth and survival of vortices in discs with radial entropy gradients and imposed cooling, finding that these discs are unstable to a finite-amplitude instability that leads to the formation of long-lived vortices - the SBI.

The SBI is a non-linear convective instability that leads to the formation and amplification of vortices when the radial stratification satisfies the Schwarschild instability criterion:

$N_{R}^{2}<0$

and when the flow undergoes thermal relaxation. $N_{R}$ is the radial Brunt Vaisala frequency and is defined by

$N_{R}^{2}=-\frac{1}{C_{\mathrm{p}} \rho} \frac{\partial P}{\partial R} \frac{\partial S}{\partial R}$

where $\rho$ is the density, $P$ is the pressure, $S=C_{\mathrm{p}} \ln \left(P^{1 / \gamma} \rho^{-1}\right)$ is the entropy per unit mass, and $C_{\mathrm{p}}$ is the specific heat capacity at constant pressure. The thermal relaxation time scale must be of the order of one orbital period. Too short a time scale prevents vortex formation, while too long a time scale does not allow vortices to be amplified. When the stratification is stable $\left(N_{R}^{2}>0\right)$ vortices are observed to form, but instead of being amplified they decay and dissolve into the background flow. The non-linear character of this instability implies that sufficiently strong perturbations are required to trigger it.

\subsection{Convective overstability}

The convective overstability is a linear instability that depends on having $N_{R}^{2}<0$ and thermal relaxation on $\sim$ dynamical time-scales (Klahr \& Hubbard 2014 Lyra 2014). The instability involves the growth of horizontal epicyclic oscillations, hence the name by which it is known, and according to the study by Lyra (2014) it leads to the formation of long lived vortices. It has been suggested as a possible source of the finite amplitude perturbations required for the SBI to operate. Vertical buoyancy has not been included in the analysis of this instability so far, so its behaviour in vertically stratified discs remains unexplored.

\subsection{VSI}

The Rayleigh criterion for hydrodynamic stability is satisfied by discs with strictly Keplerian rotation profiles because

$\frac{d j^{2}}{d R}>0$,

where $j=R^{2} \Omega(R)$ is the specific angular momentum. More generally, a disc with angular velocity varying with both radius and height, $\Omega(R, Z)$, that is subject to adiabatic, axisymmetric perturbations is stable according the Solberg Hoiland criteria (e.g. Tassoul 1978). For accretion discs with negative radial and vertical pressure gradients these stability criteria can be written

$\frac{1}{R^{3}} \frac{\partial j^{2}}{\partial R}+\frac{1}{\rho C_{\mathrm{p}}}\left(\left|\frac{\partial P}{\partial R}\right| \frac{\partial S}{\partial R}+\left|\frac{\partial P}{\partial Z}\right| \frac{\partial S}{\partial Z}\right)>0$

$\frac{\partial j^{2}}{\partial R} \frac{\partial S}{\partial Z}-\frac{\partial j^{2}}{\partial Z} \frac{\partial S}{\partial R}>0$
For a nearly inviscid disc in which perturbations are no longer adiabatic, the thermal evolution of perturbed fluid elements can remove the stabilizing influences of entropy gradients when the cooling time is short enough. This leads to the well-known Goldreich Schubert Fricke instability (Goldreich \& Schubert 1967; Fricke 1968), which in the context of accretion discs is known as the VSI (Urpin 2003). The VSI develops when the flow is vertically sheared and almost locally isothermal. The instability criterion is:

$\frac{\partial j^{2}}{\partial R}-\frac{k_{R}}{k_{Z}} \frac{\partial j^{2}}{\partial Z}<0$.

Vertical shear is always present in a protoplanetary disc, unless the flow is isothermal or homentropic, and $\left|\partial j^{2} / \partial R\right| \gg\left|\partial j^{2} / \partial Z\right|$, so the instable modes have $k_{R} \gg k_{Z}$ (radial wavelengths are much shorter than vertical wavelengths). In the locally isothermal limit, the maximum growth rate of the VSI depends on the temperature profile and the scaleheight :

$\Gamma_{\max } \sim|q|\left(\frac{H}{R}\right) \Omega$

where $q$ is the temperature profile power-law index. In a recent study, Nelson et al. (2013) showed that the VSI can cause a disc to become highly turbulent in the locally isothermal regime, with velocity perturbations having very short radial wavelengths such that there are strong local gradients in the flow. The only simulations conducted in 3D in that work utilized a locally isothermal equation of state, and the resulting turbulence led to the excitation of spiral density waves in the flow but no obvious signs of long lived vortices. It therefore remains an open question whether or not the VSI can lead to the formation of perturbations that generate vortices when thermal relaxation is not treated as being instantaneous, perhaps through the RWI or the SBI acting on the primary perturbations generated by the VSI.

\subsection{Elliptical instability}

Lesur \& Papaloizou (2009), using a local approach, showed that $3 \mathrm{D}$ elliptical vortices may be unstable. The vertical modes $\left(\vec{k}=k_{z} \overrightarrow{e_{z}}\right)$ are the dominant modes in vortices with an aspect ratio $1.5<\chi<4$, and have a growth rate given by:

$$
\Gamma=\mathcal{S} \sqrt{-\left(\frac{2 \Omega}{\mathcal{S}}-\frac{\chi}{\chi-1}\right)\left(\frac{2 \Omega}{\mathcal{S}}-\frac{1}{\chi(\chi-1)}\right)}
$$

where $\mathcal{S}$ is the shear and $\chi$ the aspect ratio of the vortex. In the case of a Keplerian protoplanetary $\operatorname{disc} \mathcal{S}=1.5 \Omega$, and the growth rate is:

$\Gamma=\frac{3}{2} \Omega \sqrt{-\left(\frac{4}{3}-\frac{\chi}{\chi-1}\right)\left(\frac{4}{3}-\frac{1}{\chi(\chi-1)}\right)}$

The fact that the mode is purely vertical and the growth rate is independent of the wavelength makes the instability quite easy to capture and very high resolution simulations are not required for it to be resolved. It should be noted that this result, however, is valid in the local approximation and is not true for long wavelengths.

The case of larger aspect ratio vortices is more complex because the instability is fully three dimensional. The 
instability is due to the resonance between inertial waves in an unstratified flow or high frequency buoyancy waves in a stratified flow and the turnover frequency of the vortex. As no inertial modes can match the turnover frequency in a vortex with $4<\chi<5.9$, these vortices are stable when the flow is not stratified, while in a stratified flow vortices are always unstable.

However, despite the unstable character of vortices in that case, the elliptical instability is difficult to observe because of the high resolution needed to resolve it. The unstable mode will have $k_{\phi}^{\max } \sim k_{z}$ and $k_{r}^{\max } \sim \chi k_{z}$, so the radial resolution needed to resolve it depends on the aspect ratio. Moreover, the growth rate for $\chi>6$ is about 50 time smaller than for vortices with $\chi<4$. These two points make the elliptical instability difficult to observe in numerical simulations that contain large aspect ratio vortices.

\subsection{Streaming instability}

Although the streaming instability applies to a disc composed of interpenetrating gas and solids, rather than to the single fluid system considered here, we discuss it briefly for completeness. It arises as a linear instability when aerodynamic drag causes inwards radial drift of solid particles, and the backreaction on the gas is included in the dynamics (Youdin \& Goodman 2005). The linearly growing modes consist of particle density enhancements with growth times that lie between fast dynamical time-scales and slower radial drift time-scales, and maximal growth rates arise for particle stopping times comparable to dynamical time-scales and when the local solids-to-gas ratio is of the order of unity. Nonlinear simulations indicate that it can lead to severe clumping of solids, such that particle concentrations are able to collapse directly to form planetesimals (Johansen et al. 2007). As such, this provides an alternative to the concentration of dust in vortices as a means of forming planetesimals in protoplanetary discs.

\section{NUMERICAL METHOD}

The equations of motion are for a compressible and inviscid fluid subject to a central gravitational potential:

$\frac{\partial \rho}{\partial t}+\vec{\nabla} \cdot(\rho \vec{V})=0$

$\frac{\partial \rho \vec{V}}{\partial t}+\vec{\nabla} \cdot(\rho \vec{V} \vec{V})=-\vec{\nabla} P-\rho \vec{\nabla} \Phi$

$\frac{\partial \rho E}{\partial t}+\vec{\nabla} \cdot \vec{V}(\rho E+P)=\rho \vec{V} \cdot \vec{\nabla} \Phi-\frac{\rho}{\gamma-1} \frac{T-T_{0}}{\tau}$.

Here $\rho$ is the density, $\vec{V}$ the velocity, $P$ the pressure and $E$ the total energy per unit mass, and $\Phi=-G M / r$ the gravitational potential due to the central star. The system is closed using the perfect gas equation of state: $\rho E=P /(\gamma-$ $1)+1 / 2 \rho \vec{V}^{2}$, where we adopt $\gamma=7 / 5$. The last term in the energy equation 13 is a thermal relaxation term: the temperature relaxes to the initial temperature $T_{0}$ with a relaxation time $\tau$. $\tau$ is assumed to be a function of $R$, the cylindrical radius, being a fixed multiple or fraction of the local Keplerian orbital period.
We adopt a radial power law for the disc temperature while assuming that the disc is initially isothermal in the vertical direction, and we also adopt a radial law for the midplane density:

$$
\begin{aligned}
T(R) & =T_{0}\left(\frac{R}{R_{0}}\right)^{q} \\
\rho(R, 0) & =\rho_{0}\left(\frac{R}{R_{0}}\right)^{p},
\end{aligned}
$$

where $R_{0}$ is a reference radius. Other quantities of interest are determined using the equations of force balance in the radial and vertical directions:

$\frac{\partial P}{\partial R}=-\frac{G M R}{r^{3}}+R \Omega^{2}$
$\frac{\partial P}{\partial Z}=-\frac{G M Z}{r^{3}}$

where $r=\sqrt{R^{2}+Z^{2}}$ is the spherical radius.

The equilibrium solutions for density and angular velocity give

$\rho(R, Z)=\rho_{0}\left(\frac{R}{R_{0}}\right)^{p} \exp \left(\frac{G M}{c_{s}^{2}}\left[\frac{1}{r}-\frac{1}{R}\right]\right)$

$\Omega(R, Z)=\Omega_{k} \sqrt{1+(p+q)\left(\frac{H}{R}\right)^{2}+q\left(1-\frac{R}{r}\right)}$

where $\Omega_{k}=\sqrt{G M / r}$ is the Keplerian velocity and $H$ is the scaleheight defined through:

$H=\frac{c_{s}}{\Omega_{k}}$.

$c_{s}$ is the isothermal sound speed defined through:

$c_{s}^{2}=\frac{P}{\rho}$,

and we define the local disc aspect ratio $h=H / R$.

In the disc model considered here, the Brunt-Vaisala frequency in the midplane is:

$N_{R}^{2}=\frac{T}{\gamma R^{2}}(p+q)(p(\gamma-1)-q)$

then the radial stratification is stable when $q<p(\gamma-1)$ and unstable otherwise.

These equations are solved in spherical coordinates $(r, \theta, \phi)$ using a finite volume code using the MUSCL Hancok method (Richard et al. 2013) We choose reflecting boundary conditions at the radial and vertical boundaries because of their ease of implementation, and because Nelson et al. (2013) have shown that the boundary conditions appear to have no effect on the development of the VSI.

The computational domains of our simulations are as follows. In radius the inner boundary is located at $r=1$ and the outer boundary at $r=1.5$, and the azimuthal domain runs between $\phi=0$ and $\phi=\pi / 4$. The meridional domain extends $\pm 5 \times h$ above and below the disc midplane. In all simulations except those with $h=0.05$, we use 500 grid cells in the radial direction. For models with $h=0.05$, we double the radial resolution and use 1000 grid cells. All runs use 300 grid cells in the azimuthal direction and 200 cells in the meridional direction. The equilibrium state is perturbed by adding $10^{-6} c_{s}$ amplitude white noise to each component of the velocity in all simulations presented in this paper. 


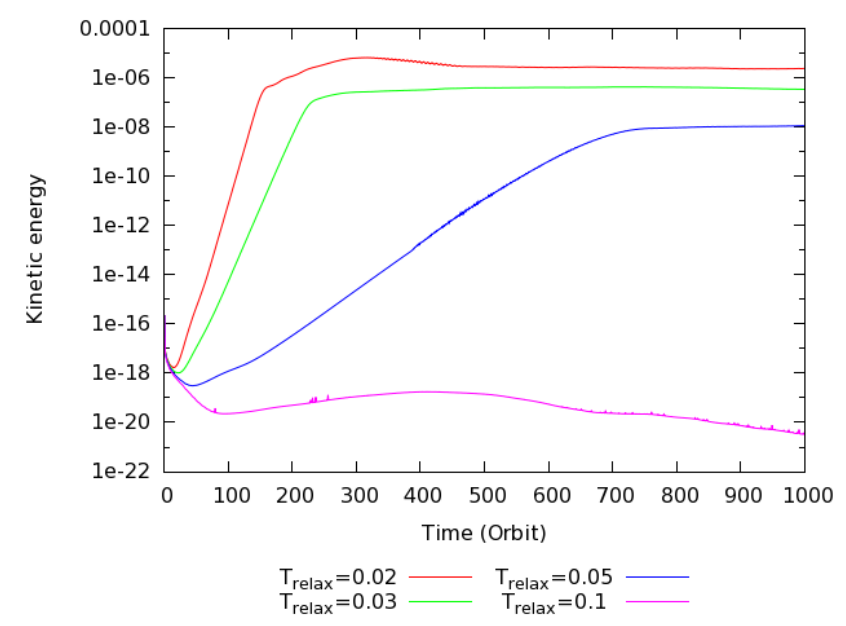

Figure 1. Kinetic energy for a disc with a $h / r=0.05$ and different values of the cooling time.

\section{AXISYMMETRIC SIMULATIONS}

An investigation of the potential for the VSI to generate vortices in discs clearly requires $3 \mathrm{D}$ simulations to be performed. The fact that the unstable VSI modes have radial wavelengths much shorter than vertical scales indicates that high resolution simulations are required, leading inevitably to long simulation run times. In light of this, most of the simulations presented in this paper adopted density and temperature power-law profiles $p=-1.5$ and $q=-2$, where the adoption of the steep temperature profile reduces the growth time scale for the VSI, hence allowing a suite of $3 \mathrm{D}$ simulations to be undertaken. We note that these disc models are stable according to the Solberg-Hoiland criteria given by equations (5) and (6) and have imaginary values of the radial Brunt-Vaisala frequency $N_{R}$. Depending on the adopted thermal relaxation time scale, these models may be unstable to the SBI and the convective overstability. It is therefore possible that any vorticity perturbations generated by the VSI may be amplified and sustained by the SBI, leading to long lived vortices.

Given that the parameters used for the simulations in this paper were not considered in the study presented by Nelson et al. (2013), we have undertaken a suite of 2D axisymmetric simulations to examine the growth times of the VSI in these models, and to also examine the critical cooling times that allow the VSI to operate. We consider models with $h=0.2,0.1$ and 0.05 with different thermal relaxation times.

Following Nelson et al. (2013), the total kinetic energy is defined by the volume integral of the sum of the radial and meridional kinetic energies, normalized by the volume integral of the initial azimuthal kinetic energy:

$E c=\frac{\int_{V} \rho\left(v_{\theta}^{2}+v_{r}^{2}\right) d V}{\int_{V} \rho v_{\phi_{0}}^{2} d V}$

The results of the 2D simulation are shown in Figs. 1, 2 and 3 , which display the evolution of the total kinetic energy in the disc models with $h=0.05,0.1$ and 0.2 respectively. For each value of $h$ the growth rate decreases when the cooling time increases, in agreement with Nelson et al. (2013), and for a critical value of the cooling time the disc becomes

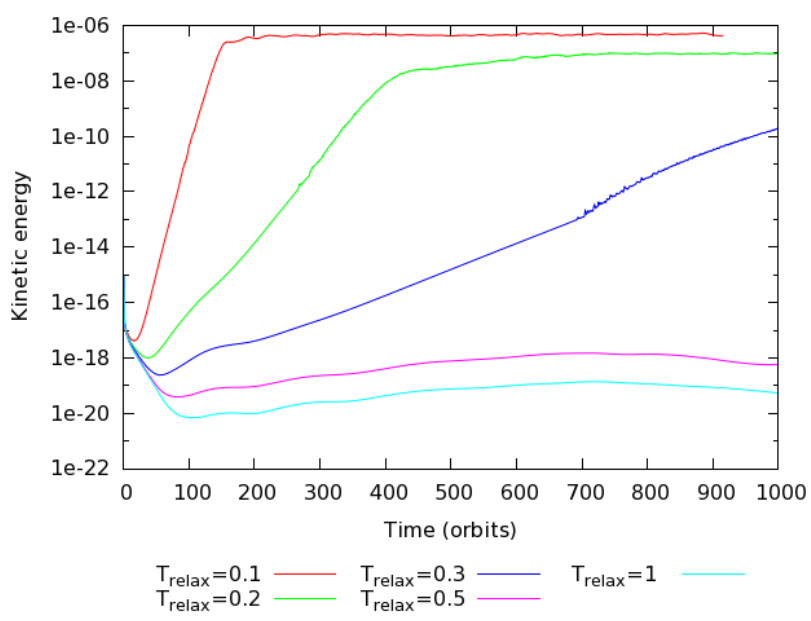

Figure 2. Kinetic energy for a disc with a $h / r=0.1$ and different values of the cooling time.

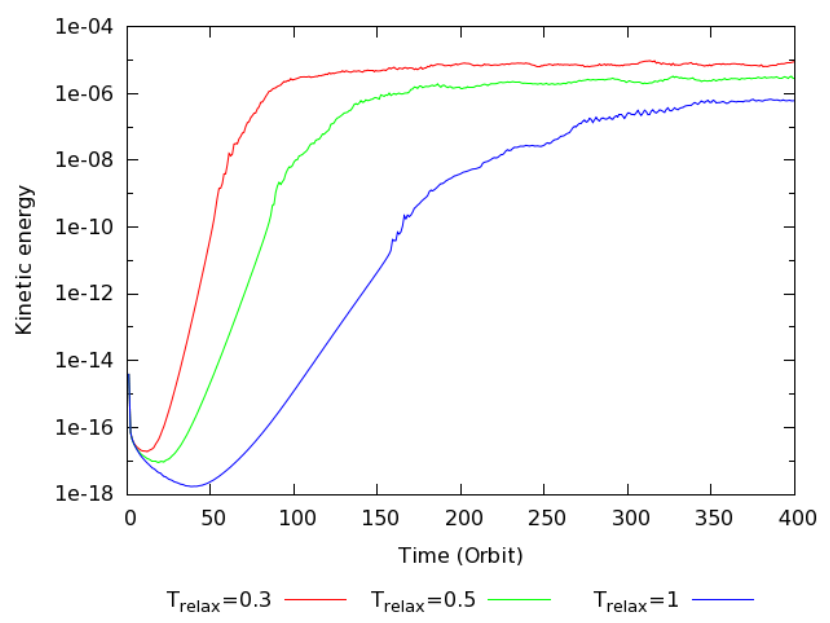

Figure 3. Kinetic energy for a disc with a $h / r=0.2$ and different values of the cooling time.

stable to the VSI. This critical value depends strongly on the scaleheight. For $h=0.05$, the disc become stable for a cooling time $0.05<\tau<0.1$, for $h=0.1$ it stabilizes for $0.3<\tau<0.5$, and for $h=0.2$ the disc remains unstable for a cooling time as large as one orbital period.

We notice that the level of saturation also depends on the cooling time: the total kinetic energy during the saturated state is higher for a shorter cooling time, and decreases for longer cooling times. In other words, higher amplitude velocity and vorticity perturbations are expected for shorter cooling times when we consider 3D simulations below.

\section{3D SIMULATIONS}

\section{$5.1 \quad$ Fiducial model}

The primary goal of this paper is to examine whether or not the nonlinear development of the VSI leads to the formation of vortices. Secondary goals include determining the range of conditions under which vortices form, understanding the nature of these vortices as a function of system parameters, 

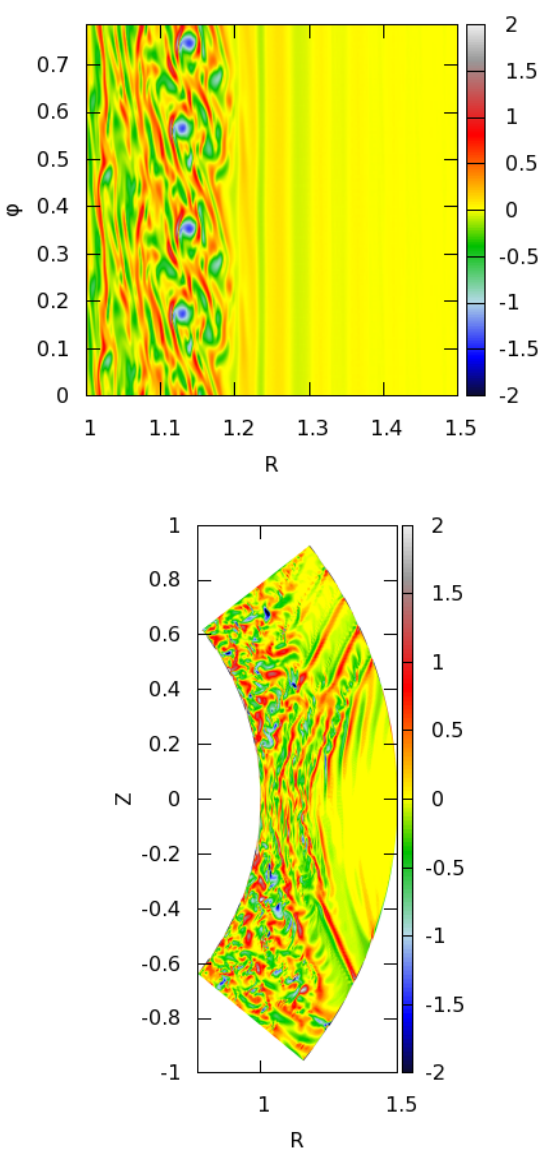
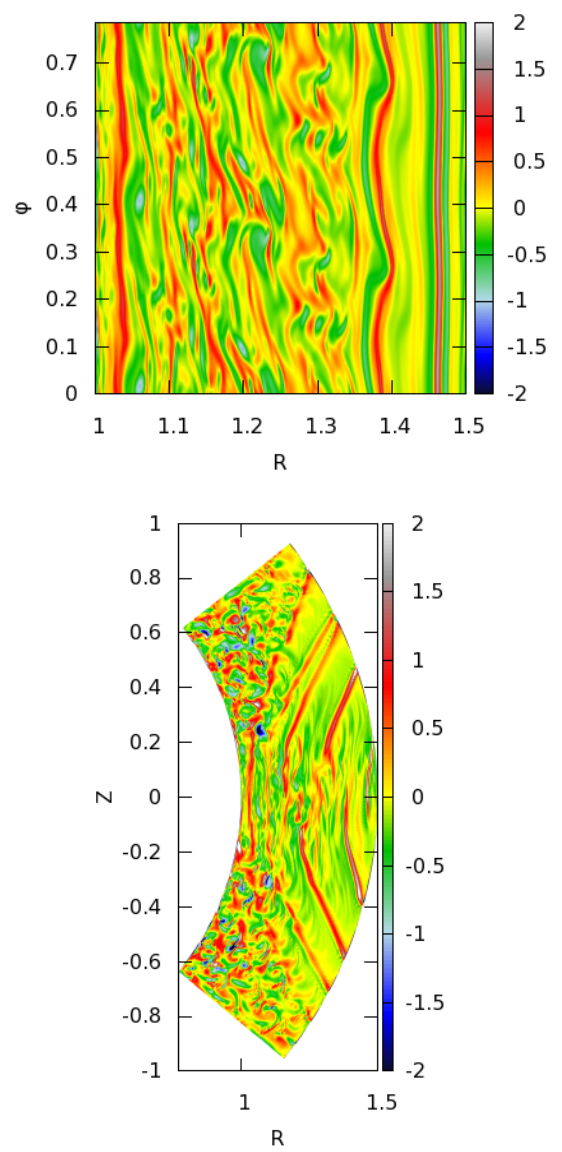
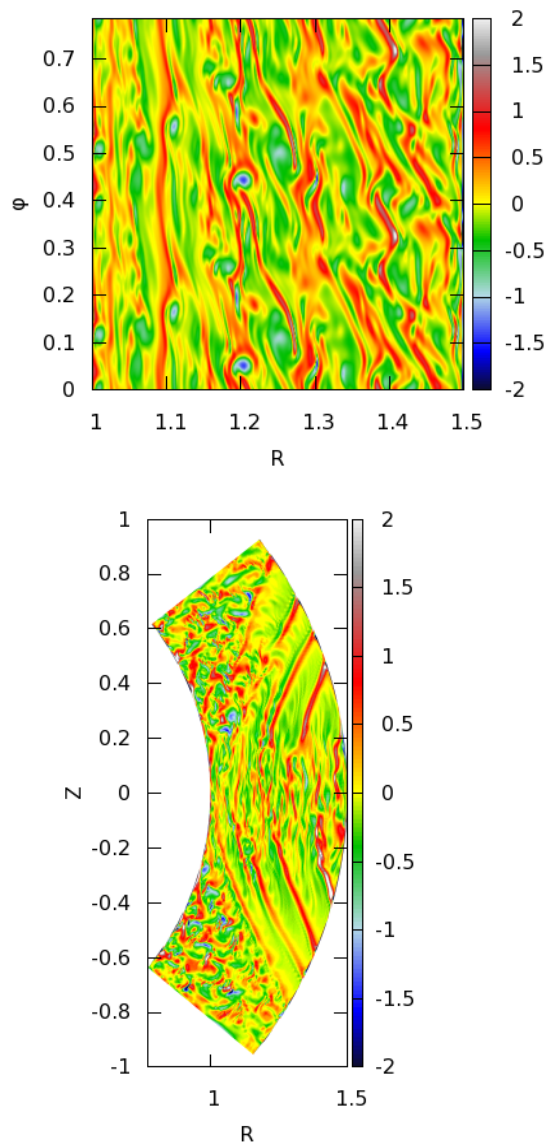

Figure 4. Vorticity profile in the midplane and meridional plane after 34,54 and 64 orbital periods, with $H / R=0.2$ and $\tau=0.1$.

and the possible roles of the RWI and the SBI in creating and maintaining vortices. Given our interest in the potential role of the SBI, we have chosen a disc model which in principle allows the development of the VSI and SBI. Both instabilities are more efficient in thick discs, so we choose $h=0.2$. Our 2D runs described in Section 4 indicate that this model remains unstable to the VSI even for relatively long cooling times, which are necessary for the SBI to operate (Lesur \& Papaloizou 2010).

Our fiducial model has a thermal relaxation time $\tau=$ 0.1 local orbits. The $2 \mathrm{D}$ simulations described in Section 4 indicate that the growth rate of the VSI should be quite large in this case, and that this cooling time is significantly shorter than the critical value for which the VSI no longer operates. During the early phases the VSI in 3D remains axisymmetric and so develops very similarly to $2 \mathrm{D}$ simulations (Nelson et al. 2013). The axisymmetric velocity perturbations correspond naturally to axisymmetric vorticity perturbations which grow with time. When these axisymmetric vorticity bands reach a critical amplitude, they tend to destabilize and vortices are formed. This evolution is illustrated by Fig. 4 which shows contours of the perturbed vertical component of the vorticity in the midplane (top panels) and in a slice along the meridional plane (bottom panels). As discussed in Nelson et al. (2013), the VSI is first observed at high latitudes in the disc and descends down towards the midplane, as seen in Fig. 4 .
The bottom panels of Fig. 4 also show that the breakup of the initially axisymmetric vorticity perturbations into discrete vortices produces structures with relatively small length scales in the vertical direction. In other words, the vortices formed in this simulation are not large scale columnar structures, but instead appear to be coherent over vertical length scales that are significantly shorter than the vertical scaleheight (i.e $\sim 0.1 H$ ). Although we only plot the vorticity in the disc midplane, we find that vortices form at all heights in the disc.

Looking in particular at the first and last of the top panels in Fig. 4, we see that the vortices have relatively small aspect ratios when projected in the $R-\phi$ plane. Detailed measurements indicate that the aspect ratio $\chi \sim 2$ for these vortices. As expected from our discussion of the elliptical instability presented in Section 2.5. these vortices do not survive for very long, and we measure a typical life time of between 2 and 3 orbits. Inspection of animations of the midplane vorticity indicates that this disc model develops a vigorous turbulent flow in which vortices continuously appear and disappear on time-scales of a few orbits.

Examination of Fig. 4 indicates that the formation mechanism of the vortices is the creation of narrow, axisymmetric vorticity perturbations that then break up into discrete vortices when the perturbation amplitude becomes large enough. From our discussion of the RWI in Section 2.1. we can see that perturbations to the vertical component 


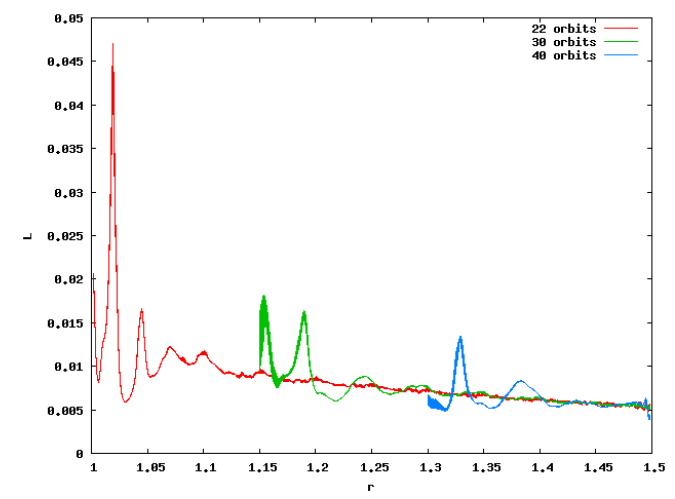

Figure 5. Profiles of the quantity $\mathcal{L}$ demonstrating the development of extrema in this quantity and hence the conditions required for the RWI to operate and form vortices.

of the vorticity that are narrowly confined in radius correspond to the creation of extrema in the quantity $\mathcal{L}$ defined by equation (1). As such, it appears that vortices form in this simulation because the VSI generates narrow vorticity perturbations and extrema in $\mathcal{L}$ that destabilize through the RWI. The SBI does not appear to play an important role in this particular simulation.

Regarding the mechanism of roll-up observed in our simulations, the axisymmetric VSI is accompanied by radial azimuthally symmetric pressure perturbations which induces jets in the zonal (azimuthal) direction. Such jets are characterized by narrow abutting azimuthal strips of positive/negative vertical vorticity (recall vorticity anomalies are here understood to be with respect to the background Keplerian frame). When the amplitude of the jet gets large enough, the RWI induces the roll-up of the negative vorticity strip leaving the positive vorticity strip more or less intact since positive vorticity anomalies in non self-gravitating discs are stable to the RWI (Umurhan 2010). An examination of the top row of Fig.s in Fig. 4 shows exactly this pattern, where the negative vorticity anomalies created by the VSI eventually roll up into localized vorticity while leaving the positive vorticity strips alone. The profile of $\mathcal{L}$ at different times during this simulation is shown in Fig. 5 , illustrating the development of the secondary RWI as caused by the VSI throughout many stages and radial locations of the simulation.

\subsection{Dependence on viscosity}

In this section we investigate the role of viscosity in the development of the instability. We have performed several simulations using the same disc model described in the previous section, but adding viscous stresses and varying the kinematic viscosity, $\nu$. Fig. 6 shows the growth of the instability for different values of $\nu$. As expected, it shows that the viscosity has a stabilizing effect on the VSI. The growth rate increases when the viscosity decreases until $\nu=2.5 \times 10^{-8}$, for which the behaviour is close to the inviscid case. A large viscosity totally inhibits the development of the VSI. The vorticity contours for the different viscosities are plotted in Fig. 7 after simulation run times of 26 orbits. For $\nu=2.5 \times 10^{-6}$ the flow is still axisymmetric and the vortices have not yet formed, while for the lower viscosity

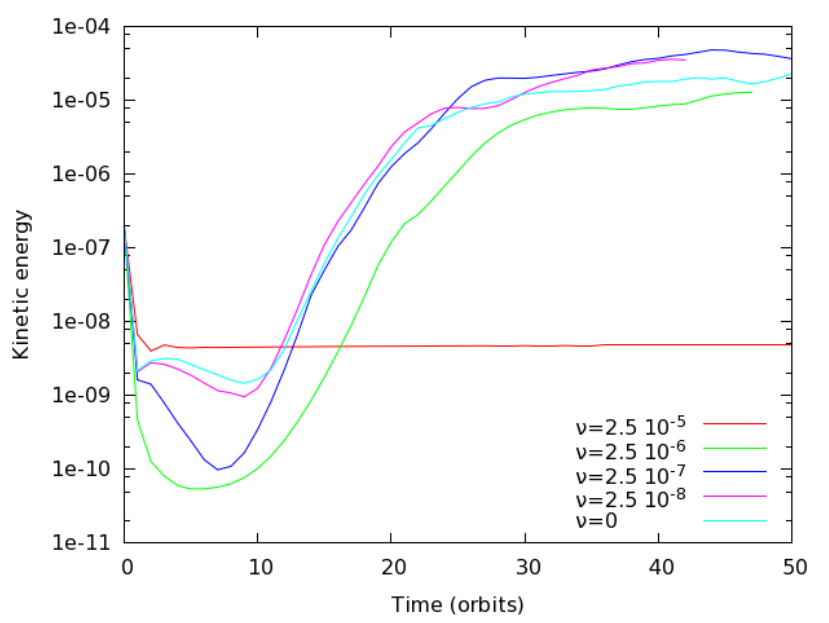

Figure 6. Kinetic energy for a disc with a $h / r=0.2, \tau=0.1$ and different viscosity.

$\left(\nu=2.5 \times 10^{-8}\right)$ the vorticity profile is very similar to the inviscid case. This suggests that the numerical diffusion is of the order of $10^{-8}$. This value correspond to a Reynolds number $R e=H c_{s} / \nu \approx 10^{6}$ and a Shakura-Sunyaev viscous stress parameter $\alpha=2.5 \times 10^{-7}$ (Shakura \& Sunyaev 1973). This value is too small to be responsible of the short lifetimes of the vortices, so we are confident that the disappearance of the vortices has a physical origin rather than a numerical one.

\subsection{Dependence on cooling time}

In this section, we investigate how vortex formation depends on the thermal relaxation by considering the cooling times $\tau=0.05$ and 0.5 , while keeping all other disc parameters the same. Inspection of Fig. 3 shows that the $\tau=0.05$ run should lead to rapid growth of the VSI, and the generation of relatively large velocity of vorticity perturbations. A cooling time of $\tau=0.5$ should lead to a longer growth time for the VSI and weaker velocity and vorticity perturbations.

Fig. 8 shows contours of the vertical vorticity perturbations for the $\tau=0.05$ run. Comparing the spectrum bars for the contours shown in Fig. 4 and 8 shows that the latter run generates significantly larger vorticity perturbations. The consequence of this is that the vortices formed in the nonlinear saturated state of this simulation have smaller aspect ratios, $\chi$, and shorter lifetimes. Close inspection of the top panels in Fig. 8 and of sequences of snapshots similar to these panels indicates that $\chi \sim 1.5$ for this run, with vortex lifetimes being approximately one orbit.

Fig. 9 show the results of the simulations with $\tau=0.5$. Inspection of the spectrum bar that indicates the amplitude of the vorticity perturbations shows that this run produces significantly weaker vorticity perturbations than the runs with $\tau=0.1$ and 0.05 . Consequently, the nature of the flow is very different in this case, consisting of elongated and longlived vortices. We estimate that the typical vortex aspect ratio in this run is $\chi \sim 6$ and the average life time exceeds the simulation run times. Close inspection of the vortices indicates that they maintain a turbulent core throughout the evolution, presumably due to the elliptical instability oper- 

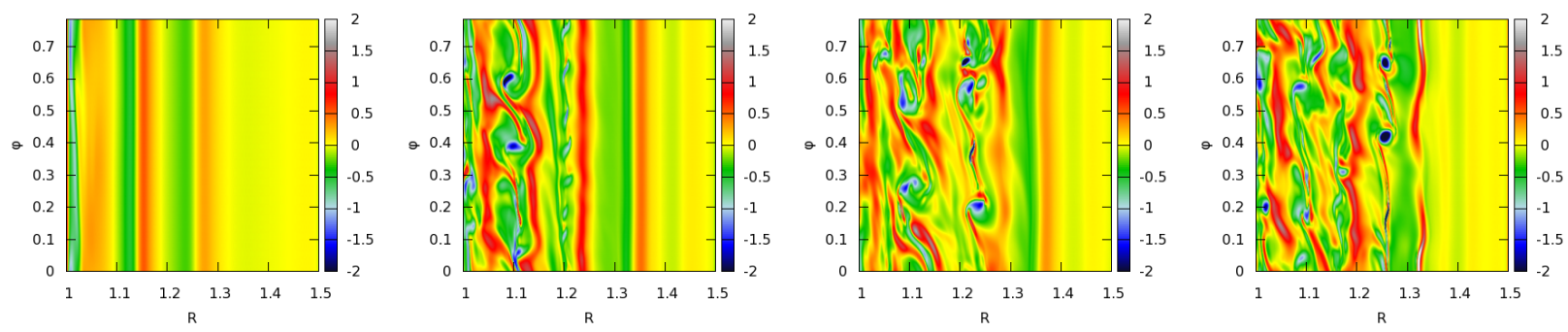

Figure 7. Vorticity in the midplane after 26 orbits and for $\nu=2.5 \times 10^{-6}, 2.5 \times 10^{-7}, 2.5 \times 10^{-8}$ and 0 .
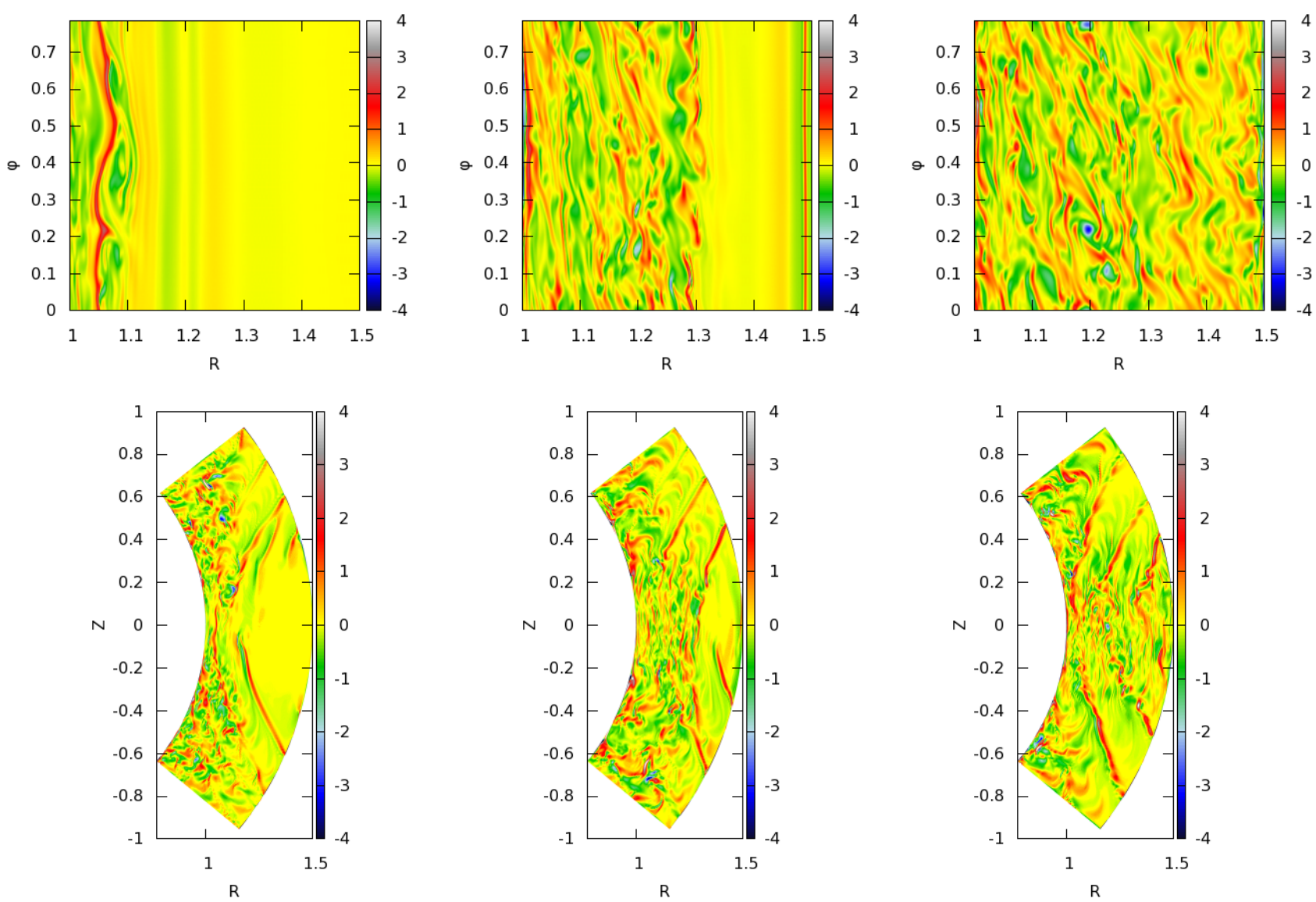

Figure 8. Vorticity contours in the midplane and meridional plane after 33, 41 and 74 orbital periods, with $h=0.2$ and $\tau=0.05$.

ating in this case. The long-lived nature of these vortices suggests that their survival is due to the action of the SBI, that continuously attempts to increase the amplitude of the vortices in opposition to the elliptical instability which attempts to destroy them. Note, however, that this statement is somewhat speculative since we may not be able to fully resolve the elliptical instability in this case.

Examining the vertical structure of the vortices in this case, we note that they occupy a greater height in the disc than those observed in the runs with $\tau=0.1$ and 0.05 . The vortices shown in the upper panels of Fig. 9 extend above and below the midplane by approximately one scaleheight.

In summary, we find that the VSI gives rise to relatively large amplitude axisymmetric vorticity perturbations when the cooling time is short (i.e. $\tau \leqslant 0.1$ orbits), and this leads to the formation of small aspect ratio $(\chi \leqslant 2)$ vortices that form through the RWI and which extend only a small distance in height (approximately $10 \%$ of the local scaleheight). The lifetimes of these vortices is found to be very short, being of the order of a few orbital periods. A longer cooling time of $\tau=0.5$ orbits gives rise to lower amplitude vorticity perturbations, leading to the formation of elongated (i.e. $\chi \sim 6$ ) vortices that extend in height by approximately 2 scaleheights and have lifetimes that exceed the simulation run times. These vortices are observed to have turbulent cores, presumably due to the elliptical instability, suggesting that their long lifetimes are due to the action of the SBI maintaining the integrity of these structures. Table 1 summarize the growth rate of the instability and the vor- 

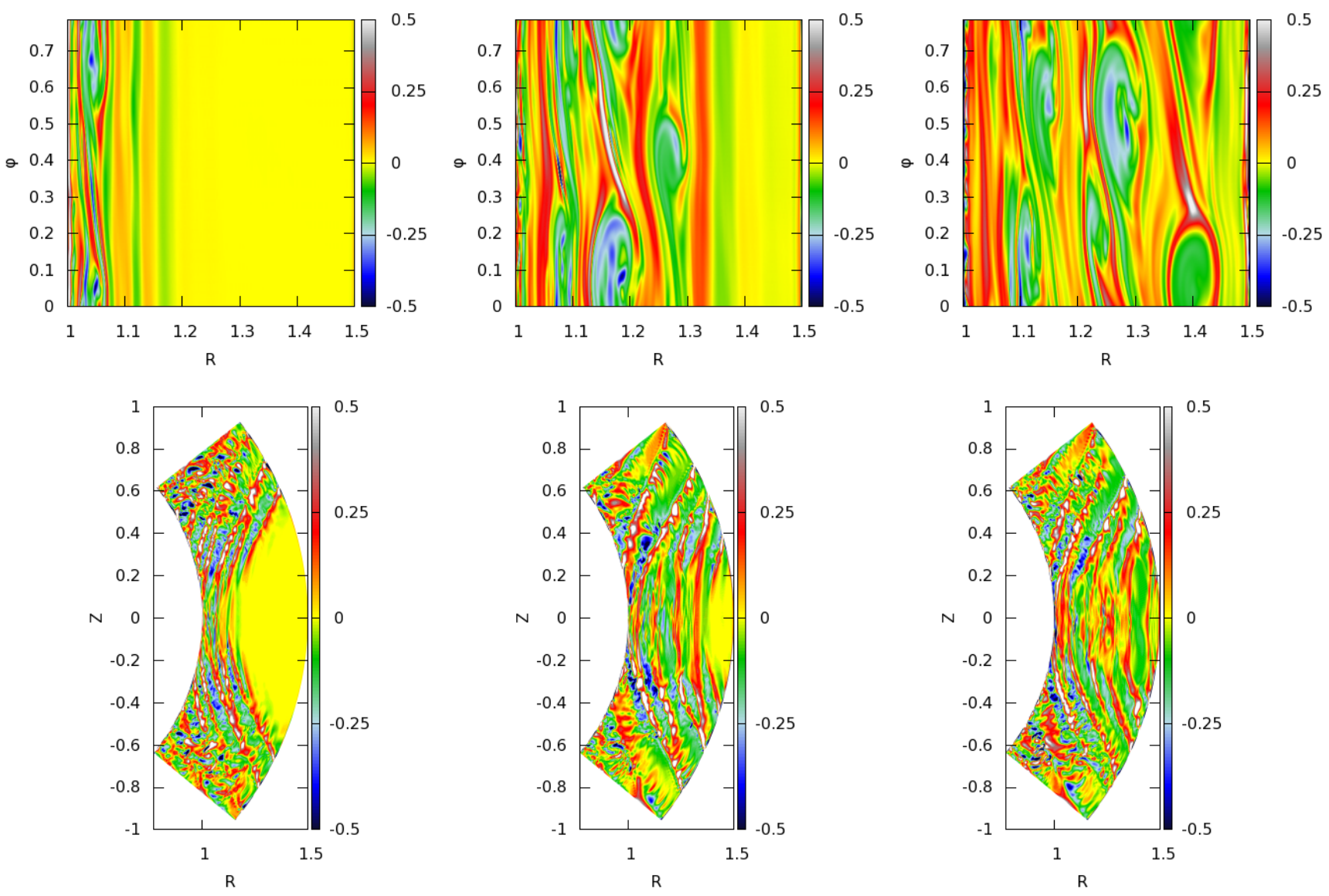

Figure 9. Vorticity profile in the midplane and meridional plane after 201, 317 and 401 orbital periods, with $h=0.2$ and $\tau=0.5$.

\begin{tabular}{cccc}
\hline $\begin{array}{c}\text { Cooling time } \\
\text { (orbits) }\end{array}$ & $\begin{array}{c}\text { Growth rate } \\
\text { (orbits) }\end{array}$ & $\begin{array}{c}\text { Vorticity } \\
\omega_{z} / \Omega\end{array}$ & Vortex aspect ratio \\
\hline 0.5 & 0.31 & -0.3 & 6 \\
0.1 & 0.9 & -1.1 & 2 \\
0.05 & 1.7 & -3 & 1.5 \\
\hline
\end{tabular}

Table 1. Results for the $h=0.2, q=-2, p=-1.5$ simulations. First column shows cooling time, second column shows growth rate of the VSI, third column shows peak vorticity perturbation (in units of the local orbital angular velocity), and fourth column shows mean aspect ratio of the emerging vortices.

ticity and the aspect ratio of the resulting vortices for each simulations.

\subsection{Dependence on scaleheight}

In the previous simulations the scaleheight was set to $h=$ 0.2 , which corresponds to a rather thick disc that may be unrealistic for a typical protoplanetary disc. Here, we investigate the evolution of two thinner disc models. As shown in Section 4, for thinner discs we need shorter cooling times to trigger the VSI. We present a simulation with $h=0.1$ and $\tau=0.2$. Inspection of the results described in Section 4 indicates that the growth time of the VSI in this case will be quite long as the cooling time is relatively close to the critical value for which the VSI switches off. Thus, we might reasonably expect behaviour that is similar to that shown by the run with $h=0.2$ and $\tau=0.5$ in this case. We also present a simulation with $h=0.05$ and $\tau=0.01$. The results presented in Section 4 indicate that this model should experience rapid growth of the VSI as the cooling time is much shorter than the critical thermal relaxation time scale. We might reasonably expect results similar to those observed for the runs with $h=0.2$ and $\tau=0.1$ and 0.05 in this case. In order to resolve the smaller length scale features expected in this run the simulation was conducted with double the resolution in the radial direction.

The results of the simulation with $h=0.1$ and $\tau=0.2$ are shown in Fig. 10. As expected, the growth time of the instability is long in this case and the vorticity perturbations that arise are of relatively low amplitude. The vortices that form are observed to have aspect ratios $\chi \sim 10$, smooth non-turbulent cores, and lifetimes that exceed the simulation run time of 695 orbits. The vertical heights of the vortices formed near the disc midplane again appear to extend approximately plus-and-minus one scaleheight about the midplane, as observed for the long-lived vortices formed in the run with $h=0.2$ and $\tau=0.5$.

The results for the simulation with $h=0.05$ and $\tau=$ 0.01 are shown in Fig. 11 . The short growth time of the VSI leads to the formation of vortices with small aspect ratios $\chi \sim 1.5$ with lifetimes of the order of one orbital period. 

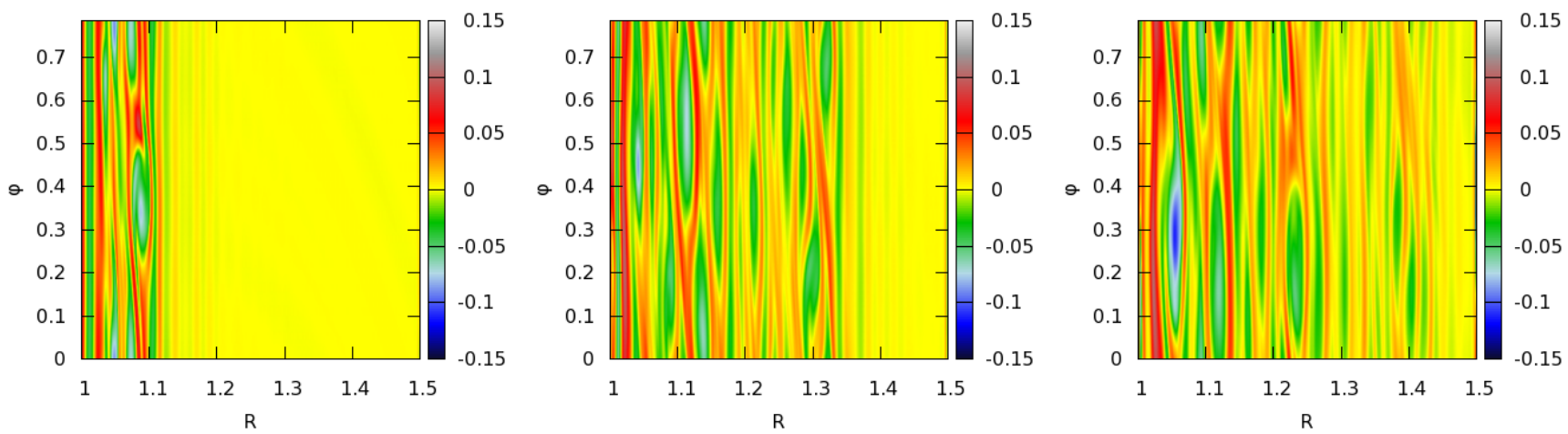

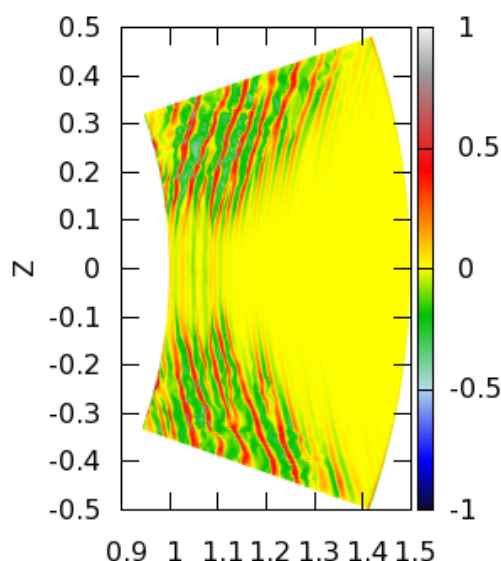

$\mathrm{R}$

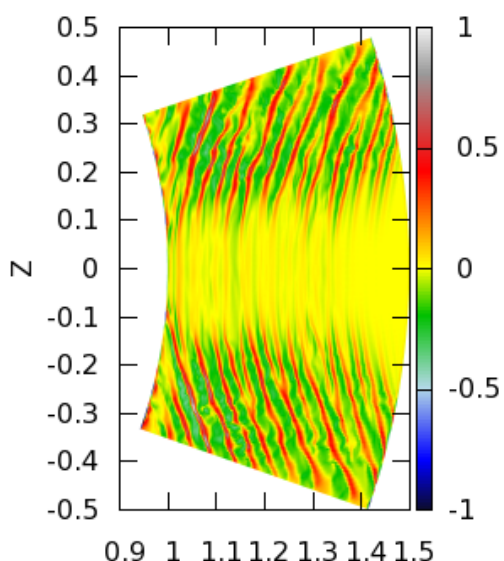

$\mathrm{R}$

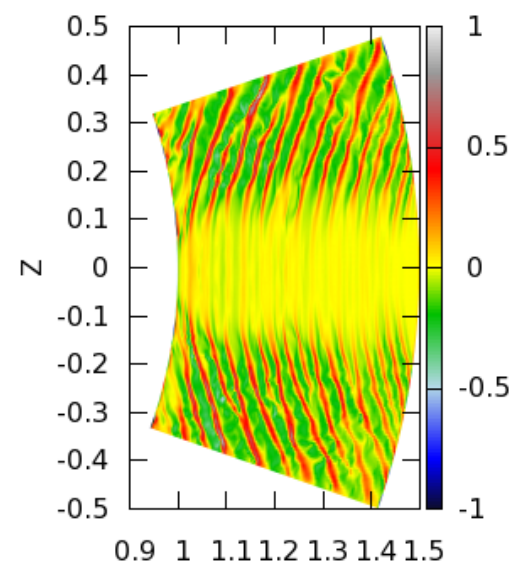

$\mathrm{R}$

Figure 10. Vorticity profile in the midplane and meridional plane after 331, 555 and 694 orbital periods, with $h=0.1$ and $\tau=0.2$.
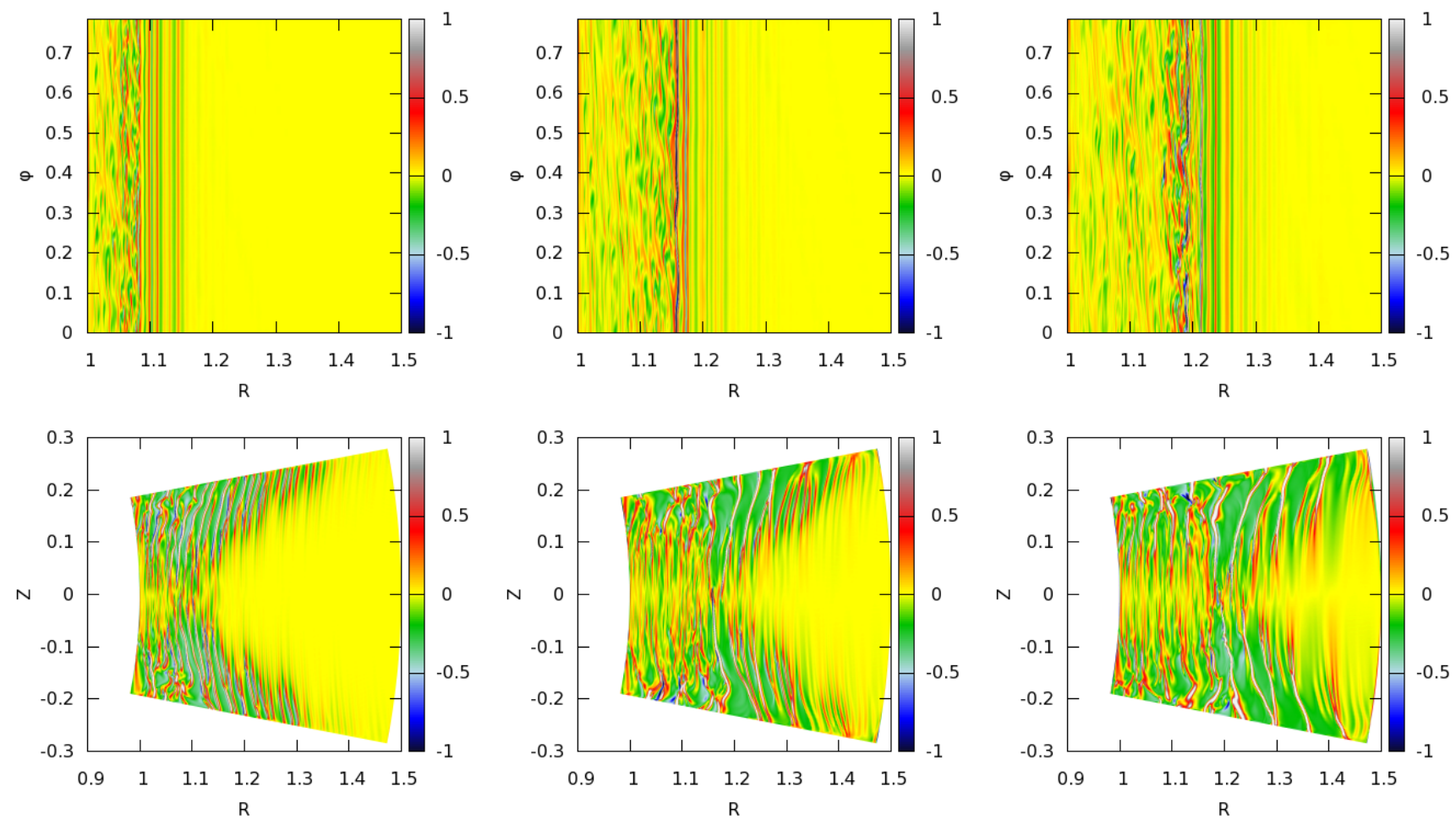

Figure 11. Vorticity profile in the midplane and meridional plane after 100, 120 and 130 orbital periods, with $h=0.05$ and $\tau=0.01$. 
Vertical shear instability and vortices

\subsection{Positive entropy gradient}

One of the original goals of this paper was to see if the VSI could produce strong enough vorticity perturbations to trigger the SBI. In their study of vortex growth in astrophysical discs, Petersen et al. (2007) showed that vortices can form in the presence of applied cooling, whether or not the radial entropy stratification was stable or unstable. They also showed that vortices are amplified and maintained over long time scales when the stratification is unstable, but decay when the stratification is stable. They found that vortex amplification is more efficient for short cooling time, but vortex formation through the SBI is more efficient if the cooling time is long.

In the previous simulations, we observed the formation of vortices even for cooling times as short as $\tau=0.01$. As discussed earlier in the paper, vortices appear to form from the axisymmetric perturbations to the vorticity that are generated by the VSI, indicating that vortex formation occurs as a secondary process driven by the RWI acting on the perturbations generated by the VSI. This suggests that the SBI is not responsible for vortex formation, but may nonetheless play a role in amplifying and maintaining long lived vortices in the flow if appropriate conditions are present in the disc.

In an attempt to clarify this point, we present two simulations that have either a positive or negative midplane entropy gradient, respectively. The model with a positive entropy gradient has disc parameters $h=0.2, q=-1, p=-3$ and $\tau=0.1$ (note the shallower temperature profile). Fig. 12 shows the evolution of the vorticity in this simulation. The results of this run are very similar to those described earlier in the paper (which all had negative entropy gradients in the midplane): the VSI develops axisymmetrically, and vortices are formed from the band of perturbed vorticity that is created by the VSI. We find that these vortices have quite large aspect ratios $(\chi \sim 8)$, but the vortex lifetimes measured in the simulation are typically in the range 15-20 orbital periods, considerably shorter than those measured in the runs discussed earlier in this paper where large aspect ratio vortices were formed.

The simulation with a negative midplane entropy gradient that was run to compare with the previous run with positive entropy gradient had identical parameters $(h=0.2$, $q=-1, \tau=0.1$ ) except for the midplane density power law which was set to $p=-2$. Nelson et al. (2013) showed that the density power law index makes essentially no difference to the growth rate of the VSI, so we expect the VSI to develop in much the same way during the early stages of these two runs. This is exactly what we observe, and in fact the long term outcome of the simulation is very similar to the one with positive entropy gradient: vortices with aspect ratios $\chi \sim 8$ are formed, and these live for approximately 15 orbital periods before dissolving into that background flow. This result indicates that for this particular set of disc parameters, the SBI has essentially no influence on the formation or amplification of vortices.

The results obtained in this section are somewhat puzzling as it is to be expected that the SBI should cause the amplification of the vortices that are formed by the VSI. Furthermore, the run described earlier in Section 5.3 with $h=0.2, p=-1.5, q=-2$ and $\tau=0.5$ showed evidence that vortices formed by the VSI were maintained against decay by the action of the SBI. In order to explore this issue further, we allowed the simulation with negative entropy gradient described earlier in this section to evolve until it had reached a saturated state, and we then restarted it with longer cooling times (both $\tau=0.5$ and $\tau=1$ ). In both cases we observed that the vortices decayed and the VSI stopped operating, such that the disc evolved towards a laminar state. This suggests that either the cooling time scale needs to be fine-tuned in order to observe the SBI in this model, or perhaps a more likely explanation is that the disc model with $h=0.2, p=-1.5, q=-2$ and $\tau=0.5$ has a steeper entropy gradient which allows the SBI to operate more strongly in that disc, whereas in a disc with weaker entropy gradient the SBI at best operates very weakly and has little influence on the formation and lifetimes of vortices.

\subsection{Disc with more realistic density and temperature profiles}

In this section, we investigate what might be thought of as a disc with a more realistic density and temperature profile than the previous models. We adopt $p=-1.5, q=-1$, $h=0.05$ and $\tau=0.01$.

In the previous simulations the parameters of the disc were chosen to favour rapid growth of the instability. The temperature power law was set to $q=-2$ because the growth rate of the VSI is proportional to $|q|$, and it gives a strong negative entropy gradient in the midplane. The scaleheight was set to $h=0.2$ to obtain faster growth of the VSI and to increase the entropy gradient. These parameters also lead to a disc that is unstable to the VSI with a cooling time that is long enough to also allow the development of the SBI. Our previous simulations, however, show that vortices more likely form due to the RWI rather than the SBI, so a strong entropy gradient and long cooling time is not necessary to form vortices.

Fig. 13 shows the result of the simulation with $h=0.05$, $p=-1.5, q=-1$ and $\tau=0.01$. As for the previous simulations with a thin disc, the radial resolution is doubled from $N_{r}=500$ to $N_{r}=1000$. As usual, the VSI develops axisymmetrically. When the rings of vorticity destabilize, they give rise to vortices that survive for approximately two orbits and the disc displays small scale turbulence. This turbulence tends to decay and after a few orbits the disc relaminarizes. Then new VSI modes start to grow, and the process resumes, resulting in intermittent behaviour in which turbulence and small scale vortices regularly appear and disappear.

\subsection{Transport properties}

We now investigate the accretion rate resulting from the turbulence generated by the VSI. We defined the Reynolds stress through:

$T_{r}(r, \theta)=\frac{1}{\phi_{\max }} \int_{0}^{\phi_{\max }} \rho \delta v_{r} \delta v_{\phi} d \phi$

where $\delta v_{r}$ and $\delta v_{\phi}$ correspond to the local radial and azimuthal velocity fluctuations:

$\delta v_{r}=v_{r}-\left\langle v_{r}\right\rangle_{\phi}$

$\delta v_{\phi}=v_{\phi}-\left\langle v_{\phi}\right\rangle_{\phi}$. 

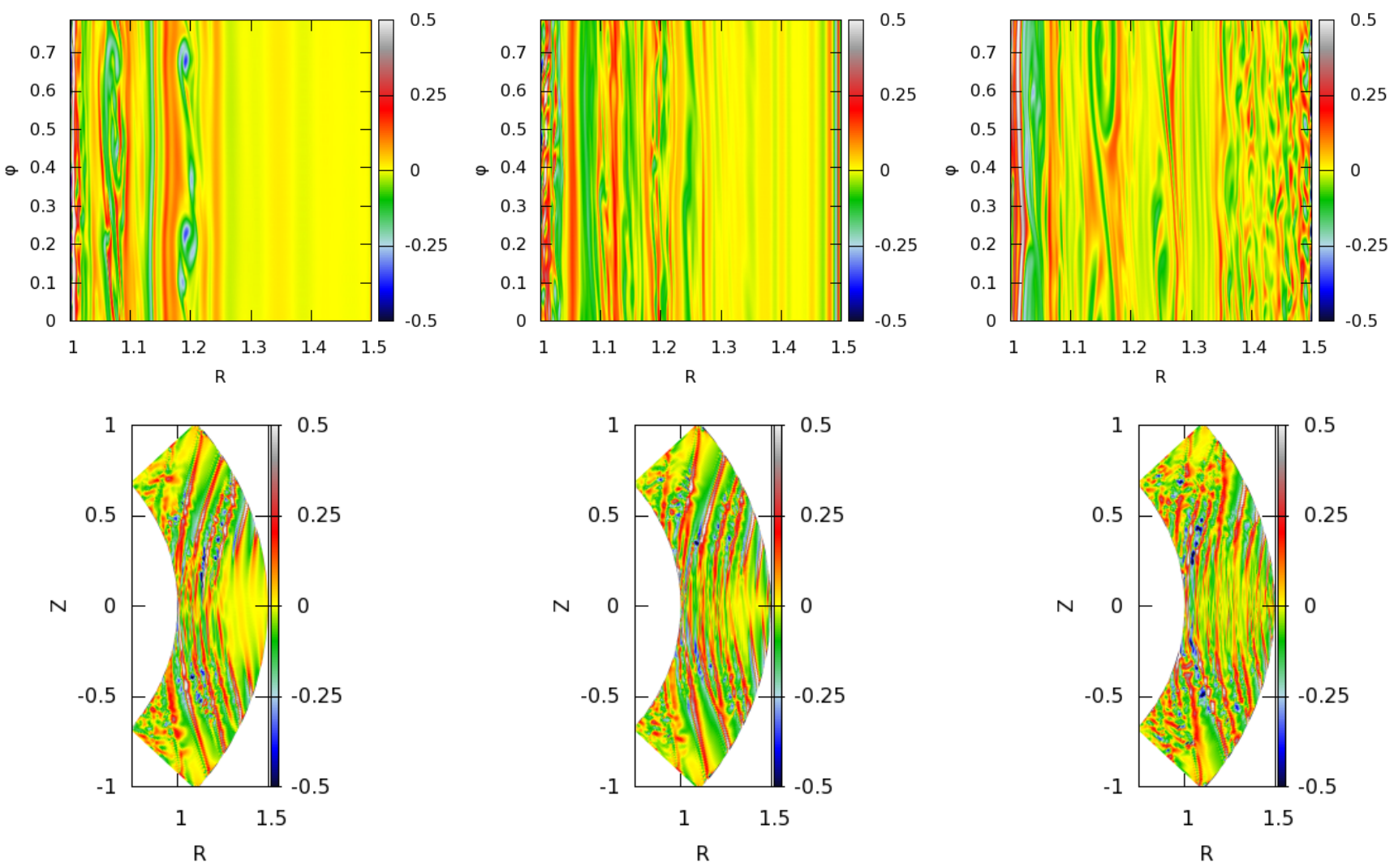

Figure 12. Vorticity profile in the midplane and meridional plane after 207, 237 and 295 orbital periods, in a disc with a positive entropy gradient in the midplane.

\begin{tabular}{ccccc}
\hline$\tau$ & $h$ & $q$ & $p$ & $\alpha$ \\
\hline 0.05 & 0.2 & -2 & -1.5 & $2.65 \times 10^{-4}$ \\
0.1 & 0.2 & -2 & -1.5 & $5.33 \times 10^{-5}$ \\
0.5 & 0.2 & -2 & -1.5 & $2.8 \times 10^{-5}$ \\
0.2 & 0.1 & -2 & -1.5 & $2.8 \times 10^{-6}$ \\
0.01 & 0.05 & -2 & -1.5 & \\
0.1 & 0.2 & -1 & -3 & $2.8 \times 10^{-6}$ \\
0.1 & 0.2 & -1 & -2 & $3.5 \times 10^{-6}$ \\
0.01 & 0.05 & -1 & -1.5 & \\
\hline
\end{tabular}

Table 2. The first column gives the cooling time, the second column the disc aspect ratio, the third and fourth columns the temperature and density power law indices, and the last column gives the effective viscous stress parameter $\alpha$ obtained in the simulations.

The local stress parameter $\alpha$ is obtained by dividing the Reynolds stress by the density-weighted mean pressure :

$\bar{P}(r)=\frac{\iint \rho P \sin (\theta) d \phi d \theta}{\iint \rho \sin (\theta) d \phi d \theta}$.

The volume- and time-averaged $\alpha$ value for each simulation is listed in Table 2, where the time average was taken over the last 20 orbits of each run. There is a general tendency for the Reynolds stress to decrease as the cooling time increases, due to the velocity fluctuations generated by the VSI decreasing with increasing cooling time. We also note that the simulation with $h=0.1$, which sustains smooth long-lived vortices in a relatively quiet background flow without strong turbulence has the lowest $\alpha$ value.

We also plot the temporal evolution of the mean $\alpha$ value for each of the simulations with $h=0.2, q=-2$ and $p=$ -1.5 in Fig. 14 The simulation with $\tau=0.05$ is shown in the top left panel, which displays a peak value of $\alpha=6 \times 10^{-4}$. The simulation with $\tau=0.1$ is plotted in the top right panel, and shows a peak value of $\alpha \sim 10^{-4}$. The evolution of $\alpha$ for the run with $\tau=0.5$ is shown in the bottom left panel and displays a peak value of $\alpha \sim 8 \times 10^{-5}$, with typical values $\alpha \sim 5 \times 10^{-5}$. Finally, the evolution of $\alpha$ for the run with $h=0.1, q=-2$ and $\tau=0.2$ is shown in the bottom right panel of Fig. 14 . Here we see that the smooth flow associated with this run containing long lived and high elongated vortices produces a small Reynolds stress that has a stress parameter that has typically values of $\alpha \sim 2 \times 10^{-6}$ during the simulation.

\section{DISCUSSION AND CONCLUSIONS}

We have presented a series of customized and idealized simulations to examine the nonlinear saturated state of astrophysical discs undergoing the VSI, with the focus being on the formation and evolution of vortices. The results of these simulations may be summarized as follows.

- We find that the VSI readily leads to the formation of vortices in a broad range of disc models. 

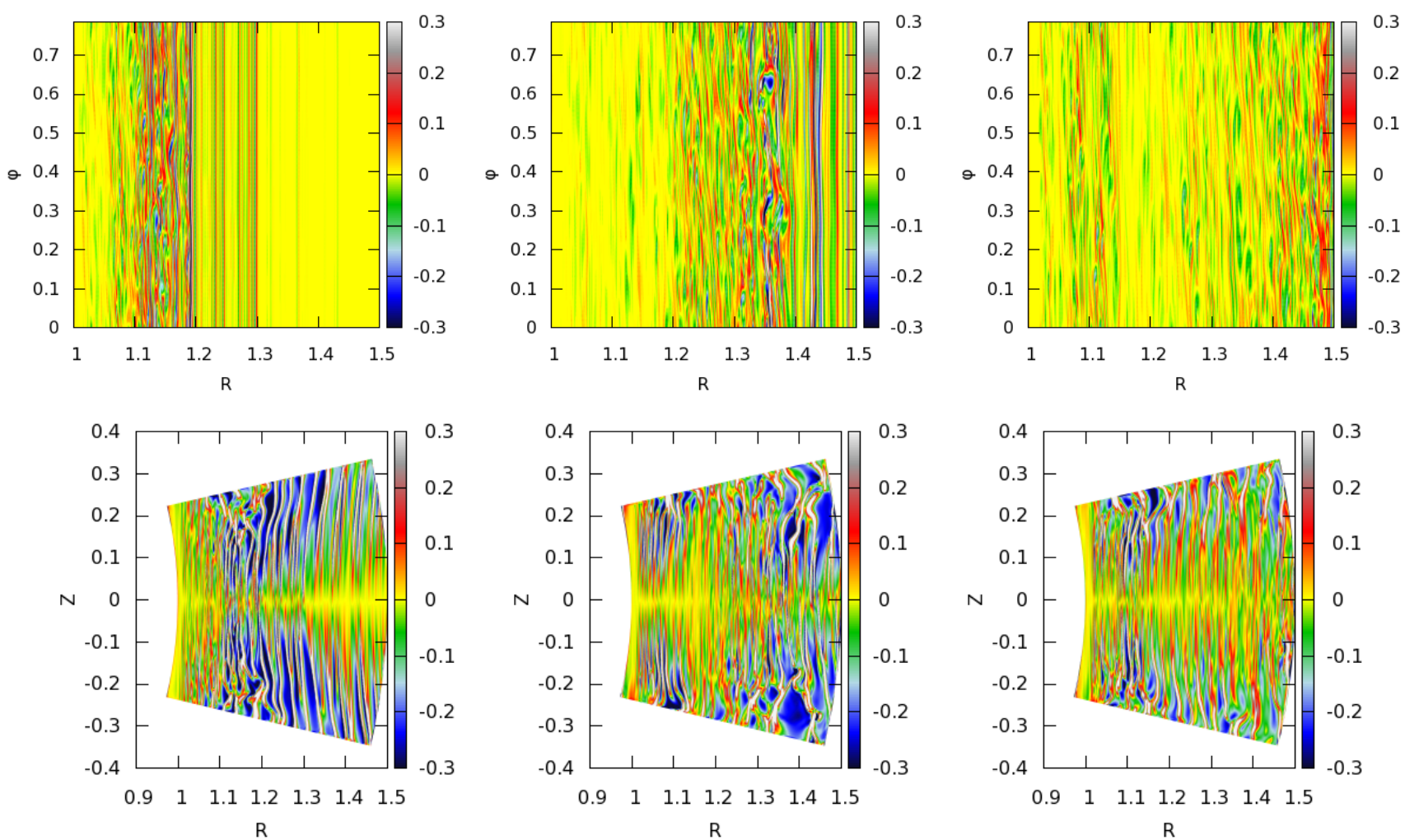

Figure 13. Vorticity profile in the midplane and meridional plane after 198,254 and 291 orbital periods, with $H / R=0.05, q=-1$ and $\tau=0.01$.

- The formation mechanism of the vortices is the generation of axisymmetric vorticity perturbations by the VSI that destabilize into discrete vortices through the RWI. Specifically speaking, the VSI generates pressure perturbations that induces the formation of narrow azimuthal jets. These jets are characterized by azimuthally symmetric strips of positive/negative vertical vorticity. As a given jet grows in amplitude, that side of it with the negative vorticity anomaly becomes unstable to the RWI and rolls-up into localized vortices while the positive anomaly remains intact since it itself is not unstable to the RWI. As a given simulation progresses and the VSI reaches out to larger radii, more and more unsteady vortices are produced eventually spreading across the totality of the computational domain.

- For steep temperature profiles and short cooling times ( $h=0.2, q=-2, \tau \leqslant 0.1$ orbits) we observe that vortices form with small aspect ratios $(\chi \leqslant 2)$ and extend in height over length scales much smaller than the local scaleheight $(\sim 0.1 H)$. These vortices have short lifetimes of only a few orbital periods before dissolving into the background flow, after which new generations of vortices form and dissolve in a repetitive cycle.

- For longer cooling times and steep temperature (and entropy) profiles ( $h=0.2, q=-2, \tau=0.5$ orbits) we observe the formation of elongated vortices $(\chi \sim 6)$ that live for hundreds of orbits (longer than the simulation run times). These vortices live with a noticeably turbulent core, indicating that there is a balance between the elliptical instability try to destroy the vortex and the SBI maintaining the vortex over long time-scales. This suggests that the SBI can play an important role in maintaining long lived vortices when the negative entropy profile is sufficiently steep.

- Similar behaviour is observed for thinner discs with $h=$ 0.1 and 0.05 when the temperature profile has a steep power law index $q=-2$.

- Changing the temperature power law index to $q=-1$ results in vortices that do not live for more than a few tens of orbits at most. Simulations performed with either weakly positive or negative entropy profiles produce very similar results, indicating that the influence of the SBI is significantly diminished when the entropy profile is weaker than that obtained with $q=-2$.

- Analysis of the volume averaged Reynolds stresses associated with the VSI shows that the efficiency of angular momentum transport depends strongly on the thermal relaxation time scale. In line with our observation that the VSI generates stronger velocity fluctuations for short cooling times, we obtain an effective Shakura-Sunyaev $\alpha$ value (Shakura \& Sunyaev 1973) of $\alpha_{\mathrm{SS}} \sim 2 \times 10^{-4}$ when $\tau=0.05$ for a model with $q=-2$ and $h=0.2$. When $\tau=0.5$ we obtain $\alpha_{\mathrm{SS}} \sim 3 \times 10^{-5}$. These values should be contrasted with the value $\alpha_{\mathrm{SS}} \sim 10^{-3}$ obtained by Nelson et al. (2013) for a locally isothermal disc model, indicating that the inclusion of a finite cooling time scale significantly reduces the strength of the VSI. This point has also been noted by Stoll \& Kley (2014), who performed simulations with radiation transport and obtained $\alpha_{\mathrm{SS}} \sim 10^{-4}$.

The requirement of short cooling times for the VSI to operate indicates that it is most likely to be present in the outer regions of protoplanetary discs, between $\sim 10$ and 50 

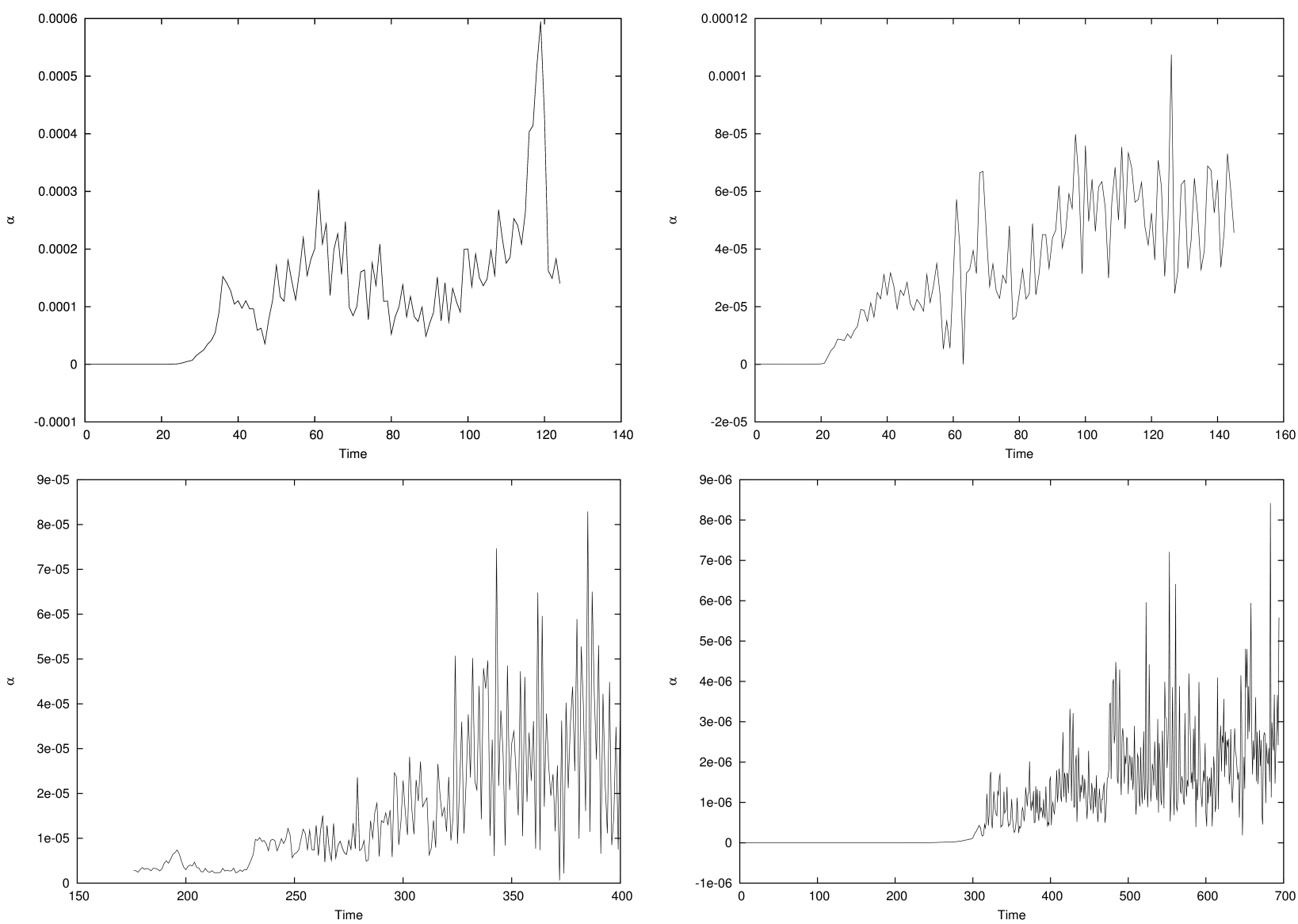

Figure 14. Mean $\alpha$ value for : a disc with a $h / r=0.2$ and $\tau=0.05$ (top left), a disc with a $h / r=0.2$ and $\tau=0.1$ (top right), a disc with a $h / r=0.2$ and $\tau=0.5$ (bottom left) and a disc with a $h / r=0.1$ and $\tau=0.2$ (bottom right).

au (Nelson et al. 2013 Umurhan et al. 2013 Lin \& Youdin 2015), although the short cooling times expected at higher disc latitudes may allow it to also operate there at smaller stellocentric radii (Lin \& Youdin 2015). The potential role of vortices in protoplanetary discs as sites for the trapping of solids and growing planets has been well documented (Barge \& Sommeria 1995), and it is of interest to address the question of whether or not the vortices formed by the VSI are likely to play an important role in planet building. The main difficulty with the VSI vortices trapping solids is their apparent short lifetimes in the absence of a sufficiently steep entropy profile. To assess the expected midplane entropy profiles in protoplanetary discs, we assume that the disc surface density profile may be written as $\Sigma(R)=\Sigma_{0} R^{\delta}$, and that the requirement for a negative entropy gradient to exist locally at the midplane (such that the SBI can play some role in extending vortex lifetimes) can be written as $q+p(1-\gamma)<0$. Then we can examine which values of $\delta$ arise for reasonable values of the temperature power-law index such that the radial Brunt-Vaisala frequency obeys $N_{R}^{2}<0$. We note that the requirement for $N_{R}^{2}<0$ can be written more conveniently as $p>q /(\gamma-1)$.

The minimum mass solar nebula model has $T(R)=$ $280 \mathrm{~K}(R / \mathrm{au})^{-1 / 2}$ (Hayashi 1981). The more sophisticated passively irradiated disc model of Chiang \& Goldreich (1997) has $T(R)=120 K(R / \mathrm{au})^{-3 / 7}$, i.e. the temperature profile is slightly shallower than the $q=-1 / 2$ value obtained from the Hayashi model. Observational constraints in the outer regions of discs indicate that $-0.7 \leqslant q \leqslant-0.4$ (Andrews \& Williams 2005), covering the theoretically expected range. Adopting $q=-1 / 2$ and $\gamma=7 / 5$, we require $p>-5 / 4$ for a negative entropy gradient at the midplane. Writing $\Sigma \sim H \rho_{\text {mid }}$, where $H$ is the scaleheight and $\rho_{\text {mid }}$ is the midplane density, and using $p=-5 / 4$ and $H(R) \propto R^{(3+q)} / 2$ (from the definition $H=c_{\mathrm{s}} / v_{\mathrm{k}}$ ), we see that the power law index for the surface density $\delta=0$ for this model. In other words, a disc that has $q=-1 / 2$ and just supports a large scale negative entropy gradient in its outer regions must have a flat or outwardly increasing surface density profile. Observations suggest that surface density power law values in the outer regions of discs are typically $\delta \sim-1$ (Williams \& Cieza 2011), such that the entropy profile is increasing and not decreasing outwards. Our simulations indicate that just having a modestly decreasing entropy profile is unlikely to be sufficient for maintaining long lived vortices, and that the entropy gradient needs to be reasonably steep. The above discussion suggests that the globally inferred temperature and surface density profiles in the outer regions of protoplanetary discs are unlikely to support the existence of long-lived vortices maintained by the SBI. We note, however, that we cannot rule out the possibility that shadowing or other effects may increase the temperature gradient locally such that vortices can exist as long-lived structures around the midplane. We note recent work studying parti- 
cle growth in evolving protoplanetary discs (Estrada et al. 2015) wherein discs with complex temperature and opacity profiles are found ones in which the VSI is likely to be active in several localized radial sections, including places near to various ice lines.

We conclude that although the VSI may operate in the outer regions of discs, and produce vortices, these are likely to be relatively short lived structures that at best play a moderate role in trapping solids and assisting in the building of planets. The presence of a quasi-turbulent flow arising from the VSI may indeed provide a source of stirring that could act to hinder the formation of planetesimals and planets (Gressel et al. 2011, 2012) or perhaps restrict it into places in the disc where the VSI is either weak or entirely absent.

Having presented a set of idealized models with simple cooling prescriptions to examine the evolution of the VSI in protoplanetary discs, the next step is to undertake multidimensional radiation-hydrodynamic simulations with realistic opacities to examine the nonlinear outcome of the VSI in more realistic disc models. These calculations will be the subject of future publications.

\section{ACKNOWLEDGEMENTS}

This work used the DiRAC Complexity system, operated by the University of Leicester IT Services, which forms part of the STFC DiRAC HPC Facility (www.dirac.ac.uk). The equipment is funded by BIS National E-Infrastructure capital grant ST/K000373/1 and STFC Operations grant $\mathrm{ST} / \mathrm{K} 0003259 / 1$. DiRAC is part of the national EInfrastructure.

\section{REFERENCES}

Andrews S. M., Williams J. P., 2005, ApJ, 631, 1134

Bai X.-N., Stone J. M., 2014, ApJ, 796, 31

Barge P., Sommeria J., 1995, A\&A, 295, L1

Barker A. J., Latter H. N., 2015, MNRAS, 450, 21

Chiang E. I., Goldreich P., 1997, ApJ, 490, 368

de Val-Borro M., Artymowicz P., D'Angelo G., Peplinski A., 2007, A\&A, 471, 1043

de Val-Borro M., Edgar R. G., Artymowicz P., Ciecielag P., Cresswell P., D'Angelo G., Delgado-Donate E. J., Dirksen G., Fromang S., Gawryszczak A., Klahr H., Kley W., Lyra W., Masset F., Mellema G., Nelson R. P., Paardekooper S.-J., Peplinski A., Pierens A., Plewa T., Rice K., Schäfer C., Speith R., 2006, MNRAS, 370, 529

Estrada P. R., Cuzzi J. N., Morgan D. A., 2015, ArXiv:1506.01420

Fricke K., 1968, ZAp, 68, 317

Fromang S., Nelson R. P., 2005, MNRAS, 364, L81

Goldreich P., Schubert G., 1967, ApJ, 150, 571

Gressel O., Nelson R. P., Turner N. J., 2011, MNRAS, 415, 3291

Gressel O., Nelson R. P., Turner N. J., 2012, MNRAS, 422, 1140

Hayashi C., 1981, Progress of Theoretical Physics Supplement, 70,35
Johansen A., Andersen A. C., Brandenburg A., 2004, A\&A, 417,361

Johansen A., Oishi J. S., Mac Low M.-M., Klahr H., Henning T., Youdin A., 2007, Nature, 448, 1022

Johansen A., Youdin A., Klahr H., 2009, ApJ, 697, 1269

Johnson B. M., Gammie C. F., 2005, ApJ, 635, 149

Johnson B. M., Gammie C. F., 2006, ApJ, 636, 63

Klahr H., 2004, ApJ, 606, 1070

Klahr H., Hubbard A., 2014, ApJ, 788, 21

Klahr H. H., Bodenheimer P., 2003, ApJ, 582, 869

Lesur G., Papaloizou J. C. B., 2009, A\&A, 498, 1

Lesur G., Papaloizou J. C. B., 2010, A\&A, 513, A60

Li H., Colgate S. A., Wendroff B., Liska R., 2001, ApJ, 551, 874

Li H., Finn J. M., Lovelace R. V. E., Colgate S. A., 2000, ApJ, 533, 1023

Lin M.-K., Youdin A. N., 2015, ApJ, 811, 17

Lovelace R. V. E., Hohlfeld R. G., 2013, MNRAS, 429, 529

Lovelace R. V. E., Li H., Colgate S. A., Nelson A. F., 1999, ApJ, 513, 805

Lyra W., 2014, ApJ, 789, 77

Lyra W., Mac Low M.-M., 2012, ApJ, 756, 62

Marcus P. S., Pei S., Jiang C.-H., Barranco J. A., Hassanzadeh P., Lecoanet D., 2015, ApJ, 808, 87

Marcus P. S., Pei S., Jiang C.-H., Hassanzadeh P., 2013, Physical Review Letters, 111, 084501

Nelson R. P., Gressel O., Umurhan O. M., 2013, MNRAS, 435, 2610

Papaloizou J. C. B., Nelson R. P., 2003, MNRAS, 339, 983

Petersen M. R., Julien K., Stewart G. R., 2007, ApJ, 658, 1236

Petersen M. R., Stewart G. R., Julien K., 2007, ApJ, 658, 1252

Richard S., Barge P., Le Dizès S., 2013, A\&A, 559, A30

Shakura N. I., Sunyaev R. A., 1973, A\&A, 24, 337

Steinacker A., Papaloizou J. C. B., 2002, ApJ, 571, 413

Stoll M. H. R., Kley W., 2014, A\&A, 572, A77

Tassoul J.-L., 1978, Theory of rotating stars

Umurhan O. M., 2010, A\&A, 521, A25

Umurhan O. M., Nelson R. P., Gressel O., 2013, in European Physical Journal Web of Conferences Vol. 46 of European Physical Journal Web of Conferences, Breathing Life Into Dead-Zones. p. 3003

Umurhan O. M., Nelson R. P., Gressel O., 2015, ArXiv:1505.01892

Urpin V., 2003, A\&A, 404, 397

Urpin V., Brandenburg A., 1998, MNRAS, 294, 399

Varnière P., Tagger M., 2006, A\&A, 446, L13

von Weizsäcker C. F., 1944, ZAp, 22, 319

Williams J. P., Cieza L. A., 2011, ARA\&A, 49, 67

Yellin-Bergovoy R., Heifetz E., Umurhan O. M., 2015, ArXiv: 1503.08470

Youdin A. N., Goodman J., 2005, ApJ, 620, 459 